\title{
FOLFIRI/Panitumumab Regimen
}

National Cancer Institute

\section{Source}

National Cancer Institute. FOLFIRI/Panitumumab Regimen. NCI Thesaurus. Code C136249.

A chemoimmunotherapy regimen consisting of leucovorin, fluorouracil and irinotecan (FOLFIRI), plus panitumumab that is used for the treatment of colon cancer. 